\title{
L'innovazione nelle piccole e medie imprese agroalimentari della Regione Campania ${ }^{1}$
}

\author{
Marcella De Martino - Fabio Magnotti - Lodovico Santoro
}

\begin{abstract}
Obiettivo del paper: Il lavoro ha l'obiettivo di analizzare i principali fattori sia interni all'azienda ( $n^{\circ}$ addetti, fatturato, competenze, investimenti in RઐS) che esterni (collaborazione, fonti informative, finanziamenti pubblici) - che favoriscono lo sviluppo di attività innovative nelle PMI del settore agroalimentare.

Metodologia: Sulla base di un framework teorico desunto dalla letteratura, è stata realizzata un'indagine empirica che ha coinvolto 122 PMI agroalimentari campane, scelte tra quelle appartenenti alle filiere pastaria, olivicola, lattiero-casearia e vitivinicola. I dati ottenuti sono stati successivamente elaborati attraverso la cluster analysis per rilevare il contributo di alcuni dei fattori alle modalità di innovazione delle PMI.

Risultati: Lanalisi ha permesso di individuare tre gruppi di imprese con differenti innovation patterns: Innovative e Collaborative (IC), Innovative e Non Collaborative (INC) e Non Innovative (NI). Dallanalisi dei dati emerge che i principali fattori discriminanti la capacità innovativa sono rappresentati dalla collaborazione con diversi stakeholder locali, dall'accesso a fonti informative e dal ricorso ai finanziamenti pubblici.

Limiti della ricerca: La realizzazione di un'indagine longitudinale di alcune best practice di piccole imprese innovative consentirebbe di approfondite il nesso di causalità tra alcuni fattori (driver) e risultati ottenuti attraverso i processi innovativi. Ulteriore limite è l'analisi delle caratteristiche dell'imprenditore quale attore del processo di cambiamento aziendale.

Implicazioni pratiche: La diffusione di "best practice" può incentivare l'adozione di pratiche collaborative da parte delle PMI afferenti ai due cluster INC e NI, anche in virtù del fatto che solitamente esse innovano per imitazione.

Originalità del lavoro: Il lavoro rappresenta un primo studio della capacità innovativa delle PMI agroalimentari della Campania. Inoltre, il lavoro ha consentito di evidenziare alcune realtà innovative che sfuggono alle rilevazioni statistiche nazionali ed europee.
\end{abstract}

Parole chiave: piccole e medie imprese; capacità di innovazione; agroalimentare

1 Il presente lavoro è stato realizzato attraverso la partecipazione al progetto di ricerca Campus innovazione QUARC: "Qualità delle produzioni tipiche campane ed il suo territorio: approcci innovativi ed integrati per rafforzare la competitività del sistema Agroalimentare", in attuazione degli obiettivi operativi 2.1 e 2.2. del Programma Operativo FESR Campania 2007/2013. 


\section{Sinergie Innovation capacity of agri-food small and medium enterprises of the Campania region}

Purpose of the paper: The purpose of this paper is to analyse the impact of some drivers - both internal ( $n^{\circ}$ employees, revenue, competences, investment in R\&D) and external (collaboration, information sources, public funding) - on the development of innovation activities in small and medium enterprises (SMEs)

Methodology: Based on a theoretical framework derived from the literature, it has been conducted an empirical analysis involving 122 agri-food SMEs of the Campania Region that belong to the grain-pasta, olive-oil, dairy and wine supply chains. The data has been elaborated through the cluster analysis technique in order to explore any meaningful patterns that may exist in terms of the innovation capacity.

Results: Three clusters with different innovation modes have been identified: Innovative and collaborative (IC); Innovative and non-collaborative (INC); Noninnovative (NI). The analysis reveals that the main discriminating factors of the innovative capacity are the collaboration with different local stakeholders the access to information sources and the ability to obtain public funding for innovation.

Research limits: A longitudinal analysis of some best practices of innovative SMEs could offer a better understanding of the relationships between some drivers and the results of the innovation. Other limits is the lack of the analysis of characteristics of the entrepreneur as the main dynamic actors of the business ri-organizational process.

Practical implications: The dissemination of "best practices" can promote the adoption of collaborative practices by SMEs of the others two clusters INC and NI, given the fact that these usually innovate by imitation.

Originality of the papaer: This is the first quantitative study focusing on the innovation capacity of agri-food SMEs in the Campania Region. The study specifically highlights the existence of some innovative SMEs that generally are overlooked by national and European statistical surveys.

Key words: small and medium enterprises; innovation capacity; agri-food chain

\section{Introduzione}

L'importanza dell'innovazione per la competitività aziendale è stata ampiamente dibattuta nella letteratura manageriale e numerosi studi hanno evidenziato come essa sia il frutto di specifiche scelte strategiche delle imprese in funzione di diverse variabili quali la dimensione aziendale (Avermaete et al., 2004; De Jong e Vermeulen, 2006), la cultura imprenditoriale (Mascitelli, 2000, Shan et al., 2016), il grado di internazionalizzazione (Boermans e Roelfsema, 2016; Özçelik et al., 2004), il livello di investimenti in attività di Ricerca e Sviluppo (Capitanio et al., 2009) e il grado di collaborazione con gli altri attori della supply chain (Kuhne et al., 2010). Tra tali variabili, quella dimensionale continua a rivestire un ruolo fondamentale nel determinare la capacità innovativa delle imprese. È infatti innegabile come la grande dimensione favorisca i processi innovativi delle imprese che possono far leva su un maggior potere contrattuale nel mercato della ricerca e del capitale intellettuale e disporre di maggiori risorse da investire in attività di Ricerca e Sviluppo $(R \& S)$ e, pertanto, svolgere con maggiore efficacia un'attività innovativa. 
Non sono mancati, tuttavia, studi da cui emerge una spiccata capacità innovativa da parte delle piccole e medie imprese, evidenziando come quest'ultima non derivi necessariamente da investimenti in attività di ricerca e sviluppo, ma possa essere alimentata da altre fonti interne ed esterne non necessariamente riconducibili ad attività formalmente misurabili (Santamaria et al., 2009). L'innovazione nelle piccole e medie imprese (PMI) è infatti spesso realizzata secondo forme, modalità e meccanismi non facilmente rilevabili, come ad esempio lo spirito creativo dellimprenditore o la creazione di rapporti collaborativi con clienti e fornitori fondati sulla fiducia (Giarretta, 2013). Per utilizzare un'espressione ricorrente e molto efficace, le piccole imprese realizzano per lo più un innovazione invisibile, nascosta, poiché sfugge alle rilevazioni statistiche ${ }^{2}$. A tal riguardo, recenti studi si sono focalizzati sul ruolo della collaborazione quale modalità di accesso a risorse esterne e a nuovi mercati, nonché come fonte di generazione di nuova conoscenza. In tal modo viene superato il limite legato alla disponibilità in-house delle risorse e delle competenze adeguate per poter avviare con successo una innovazione (Batterink et al. 2010; Colombo et al., 2012; Dagnino et al., 2015; Gronum et al., 2012).

Altri autori richiamano invece i concetti di prossimità geografica (Lundvall, 1992; Von Hippel, 1988), distretti tecnologici (Lazzeroni, 2010; Sangnuigni e De Crescenzo, 2013) e di ecosistemi dell'innovazione (Ambrosetti, 2013), al fine di evidenziare la capacità di un'area geografica di sostenere i processi di innovazione delle imprese, garantendo una più diretta circolazione delle conoscenze riguardo ai progressi tecnologici, anche attraverso meccanismi di interazione più diretti.

Tuttavia, tali interazioni sono solo parzialmente riscontrabili nel contesto italiano, specialmente nelle regioni del Mezzogiorno, dove la frammentazione del sistema produttivo ha accentuato le difficoltà, soprattutto per le PMI nel sostenere investimenti in attività di R\&S. Tale circostanza ha comportato un forte gap innovativo rispetto ai principali paesi europei (Banca d'Italia, 2012). La polverizzazione del sistema produttivo nazionale si manifesta con maggiore enfasi nel comparto agroalimentare, uno dei più rappresentativi del Made in Italy, in cui tuttavia il peso delle imprese di piccole e piccolissime dimensioni è pari a circa il $99 \%(\text { Istat, 2011) })^{3}$. In particolare, i dati del $9^{\circ}$ Censimento industria e servizi mostrano come la maggioranza $(87,1 \%)$ delle realtà produttrici del comparto agroalimentare siano microimprese con un numero di addetti minore di 10 , mentre quelle di piccole dimensioni (1049 addetti) rappresentino l'11,4\%. Considerando il forte impatto che le imprese agroalimentari rivestono sull'occupazione, si registra, inoltre, un

2 Va notato che il sistema di misurazione dell'innovazione utilizzato nelle statistiche ufficiali a livello europeo e nazionale è fondato sull'impiego di alcuni indicatori, quali la spesa in R\&S o il numero di brevetti, i quali comportano una sottostima della capacità innovativa delle imprese di piccola dimensione che innovano senza lasciarne traccia nel bilancio d'esercizio.

3 Si deve tenere presente che l'unica indagine ISTAT che coinvolge le imprese con meno di 10 addetti è rappresentata dal Censimento industria e servizi. Le indagini relative all'innovazione in Italia, seguendo le indagini europee della Community Innovation Survey - CIS, coinvolgono le imprese che hanno un numero di addetti maggiore di 10. 
sinergie Vol. 36, N. 105, 2018

valore aggiunto aggregato inferiore rispetto a quello ascrivibile alle grandi imprese.

Infine, le imprese agroalimentari italiane presentano un forte gap rispetto ai concorrenti europei, considerando aspetti legati all'innovazione. Dall'analisi dei dati europei relativi al "food and beverage" (Eurostat, 2014), risulta che il $41,7 \%$ delle imprese italiane non ha introdotto innovazioni negli ultimi 3 anni, mentre solo il 9,8\% ha sperimentato innovazioni di processo e di prodotto (contro una media europea del 13,2\%), mentre considerando le forme collaborative di innovazione solo, il 19,5\% (contro una media europea del $33,1 \%$ ) ha attivato partnership con altri soggetti.

Alla luce di tali considerazioni, il presente lavoro si pone lobiettivo di analizzare la capacità di innovazione delle piccole e medie imprese (PMI) agroalimentari campane. Adottando una visione globale del processo innovativo, il lavoro non si limita a considerare soltanto le risorse interne all'azienda ma anche quelle esterne, come lo sviluppo di forme di collaborazione con fornitori, clienti e istituti di ricerca, il ricorso a finanziamenti pubblici e l'accesso a fonti informative. Inoltre, analizzando un contesto regionale, è possibile verificare l'importanza che la prossimità geografica assume sulle scelte di innovazione delle PMI (Davenport, 2005).

Considerando come input dell'innovazione sia le risorse interne che quelle esterne all'impresa, il lavoro analizza le innovazioni delle PMI agroalimentari relativamente ai prodotti, ai processi, ai modelli organizzativi e alle strategie di marketing. I differenti percorsi di innovazione intrapresi conducono le imprese ad ottenere differenti risultati in termini di aumento del profitto, della quota di mercato, dellefficienza produttiva e della sostenibilità ambientale. Oltre a misurare la capacità delle imprese innovatrici di sfruttare le risorse interne ed esterne nei processi innovativi, vengono considerati anche i principali fattori di ostacolo incontrati dalle imprese che non presentano alcuna innovazione.

La ricerca si focalizza principalmente sulle PMI agroalimentari impegnate nella fase di trasformazione delle filiere lattiero-casearia, olivicola-olearia, cerealicola-pastaria e viticola/vitivinicola della regione Campania. Questa scelta è legata sia alla loro rilevanza socio-economica, sia all'elevata incidenza di prodotti Made in Italy provenienti da tali filiere campane. Inoltre, alle quattro filiere analizzate è riconducibile la maggior parte dei prodotti di qualità, come la Mozzarella (DOP, STG), la Pizza Napoletana (STG), la pasta di Gragnano, l'olio DOP e l'ampia gamma di vini DOP e DOCG (MIPAAF, 2016). La qualità di tali prodotti è inoltre riconosciuta anche dai mercati esteri. In particolare, secondo uno studio di European House-Ambrosetti e di Federalimentare (2018) circa il 40\% dell'export italiano è rappresentato da prodotti a denominazione protetta (DOP, IGP, ecc.) appartenenti alle filiere vitivinicole $(17,9 \%)$, lattierocasearia $(8,6 \%)$, olivicola $(6,8 \%)$ e cerealicola-pastaria $(5,8 \%)$.

Il lavoro è strutturato come segue. Il secondo paragrafo riporta l'analisi della letteratura sui nuovi modelli di innovazione nel settore agroalimentare. Nel paragrafo successivo è descritto, invece, il framework teorico impiegato per valutare la capacità innovativa delle PMI agroalimentari campane. Chiude tale paragrafo, un'attenta descrizione della struttura del questionario e della scelta del metodo statistico utilizzato nell'analisi dei dati. Nel quarto 
paragrafo vengono presentati e discussi i principali risultati emersi. A conclusione del lavoro, sono sviluppate alcune riflessioni sullo scenario in cui operano le PMI agroalimentari campane e sull'individuazione di possibili implicazioni manageriali e di policy.
Marcella De Martino Fabio Magnotti Lodovico Santoro piccole e medie impres
innovazione nelle agroalimentari della Regione Campania

\section{L'innovazione nel settore agroalimentare: drivers e modelli di analisi}

Un primo contributo rilevante per la comprensione dei modelli di innovazione delle imprese agroalimentari è offerto dal lavoro di Grunert et al. (1997) che, sulla base dell'analisi di casi studio in ambito europeo, definisce due principali driver dell'innovazione di cui il primo è rappresentato dalle attività di R\&S, che sono alla base del cambiamento tecnologico all'interno dell'impresa. Sebbene il settore sia considerato lower research intensity industry, nondimeno evidenzia un grande numero di innovazioni tese al miglioramento dei prodotti agroalimentari esistenti e/o dei relativi processi produttivi (Bareghehe et al., 2012). Il secondo driver è identificato nellorientamento al mercato, inteso come "the detection and fulfilment of unfilled needs and wants of potential customers, using the skills, resources, and competences of the company" (Grunert et al., 1997, pag. 29).

Tali drivers sono stati successivamente approfonditi da alcuni dei lavori più recenti (Avermaete et al., 2004; Acosta et al., 2015; Batterinik et al., 2006; Karantininis et al., 2010; Kuhne et al., 2010) che hanno evidenziato il ruolo determinante delle risorse interne e delle risorse esterne nellattivare processi di innovazione all'interno dell'azienda e lungo la supply chain (Tabella 1).

Nello specifico, Avermaete et al. (2004) analizzano le determinanti delle innovazioni di prodotto e di processo nelle piccole imprese agroalimentari localizzate in 6 diverse zone agricole del Regno Unito, Belgio e Irlanda. Il framework teorico proposto dagli autori si focalizza, da un lato, sulle risorse interne all'azienda quali le caratteristiche dell'imprenditore; competenze della forza lavoro e gli investimenti in know-how e dall'altro, su quelle esterne quali le consulenze e le fonti informative. Attraverso un'indagine empirica condotta su $177 \mathrm{PMI}$, gli autori identificano 4 gruppi di imprese caratterizzati da differenti modalità di innovazione, denominati rispettivamente: non-innovatrici, tradizionaliste, followers e leaders. Dai risultati, emerge come lo sviluppo delle competenze della forza lavoro risulti essere il principale driver dell'innovazione, più rilevante degli investimenti in know-how, peraltro del tutto assenti in alcune imprese del gruppo dei leaders. Questo risultato ha importanti implicazioni per le policy tese a favorire l'innovazione nelle PMI la cui efficacia, in tal caso, sarebbe certamente più significativa se orientate a favorire lo sviluppo delle competenze interne, piuttosto che rivolte genericamente a sostenere l'attività di R\&S.

Sulla base dei dati relativi a più di 300 imprese agroalimentari olandesi, Batterink et al. (2006) sottolineano l'importanza dell'orientamento al mercato, oltre che dei finanziamenti pubblici, quale driver dell'innovazione. Nonostante le evidenze emerse in letteratura circa l'importanza della 
sinergie Vol. 36, N. 105, 2018

collaborazione nei processi innovativi, gli autori non riscontrano all'interno del campione un nesso causale tra queste due variabili. Questo in conseguenza del fatto che l'unità di analisi è circoscritta a singole imprese danesi (e non alle supply chain) che hanno partecipato alla Community Innovation Survey (CIS), in cui l'analisi della collaborazione si limita esclusivamente alle innovazioni radicali di prodotto e/o processo. Molto più rilevanti risultano essere le fonti esterne di informazioni, in quanto le imprese agroalimentari sono spinte ad innovare per imitazione dei loro diretti competitors.

Le relazioni collaborative lungo la supply chain sono invece prese in considerazione nel lavoro di Kuhne et al. (2010) che, a differenza di Batterinik et al. (2006), si focalizza su 270 imprese agroalimentari appartenenti a circa 90 supply chain di prodotti tradizionali di tre paesi europei: Italia, Belgio ed Ungheria. Attraverso la cluster analysis sono stati identificati tre gruppi di imprese: il primo, formato da 31 supply chain, è caratterizzato da imprese principalmente di piccolissime dimensioni, con bassa capacità d'innovazione e con scarsa propensione alla collaborazione ed è, pertanto, definito "Non-innovator chains". Il secondo gruppo, "Non-collaborating innovator chain", è composto da 49 supply chain e si contraddistingue per una maggiore capacità innovativa rispetto al primo. Infine il terzo cluster è composto da 11 supply chain caratterizzate da un elevato livello di innovazione e da un intensa rete di collaborazioni ed è, pertanto, definito "High-collaborating innovator chains". I risultati di questo cluster evidenziano l'importanza che i processi collaborativi assumono per le imprese, in quanto dimostrano come la piccola dimensione non implichi necessariamente una bassa capacità di innovazione qualora vi siano strette collaborazioni con i membri della supply chain (clienti e fornitori).

Il tema della collaborazione e dell'integrazione verticale lungo la supply chain viene anche affrontato nel lavoro di Karantininis et al. (2010), che analizza la diversa natura dei legami tra le imprese (integrazione verticale, contratti e/o altri accordi di rete) e l'impatto che questi hanno sulle dinamiche di crescita sia delle singole imprese che dellintera supply chain. Grazie ad un'indagine empirica che ha coinvolto più di 400 imprese danesi, il lavoro fornisce un quadro molto dettagliato sullorganizzazione dellinnovazione e sugli effetti generati nellambito delle supply chain agroalimentari. La strategia di integrazione verticale risulta molto più rilevante nel perseguimento delle innovazioni di prodotto, soprattutto nei casi in cui il controllo viene esercitato dalle imprese a valle della supply chain. Ciò in virtù del fatto che le imprese posizionate a valle, hanno una maggior conoscenza delle esigenze del consumatore finale, driver fondamentale per l'innovazione di prodotto. Inoltre, il potere contrattuale dell'impresa ed il suo orientamento alle esportazioni rappresentano ulteriori fattori chiave per lattivazione di varie forme di innovazione lungo la supply chain.

Acosta et al. (2015) analizzano il ruolo delle politiche pubbliche nel favorire lo sviluppo di attività di R\&S e l'innovazione nelle imprese agroalimentari e il relativo impatto sulla produttività aziendale. Ispirandosi al modello sviluppato da Crepon et al. (1998), gli autori conducono un indagine longitudinale, dal 2008 al 2011, su circa 500 imprese agroalimentari spagnole. Il modello, opportunamente adattato al contesto 
di riferimento, considera tre tipologie di innovazione (di prodotto, di Marcella De Martino di riferimento, considera tre tipologie di innovazio Magnotti processo e organizzative) e valuta per ciascuna di esse l'impatto dei Lodovico santoro finanziamenti pubblici sulla produttività dell'impresa in termini di $\begin{aligned} & \text { Liccole e mediei imprese } \\ & \text { agroalimentari della }\end{aligned}$ "vendite per dipendente". Dall'indagine emerge che i fondi pubblici Regione Campania nazionali favoriscono gli investimenti in R\&S e contribuiscono, anche se marginalmente, allo sviluppo di innovazioni di prodotto ed organizzative. Le spese per la formazione, inoltre, risultano più elevate per le imprese che hanno realizzato più di una innovazione; per queste imprese si registra anche una maggiore produttività rispetto a quelle che hanno realizzato esclusivamente innovazioni di processo. Infine, anche la propensione alla collaborazione e l'orientamento alle esportazioni risultano essere fattori determinati per l'innovazione.

Tab. 1: Principali modelli di analisi dell'innovazione nelle imprese agroalimentari

\begin{tabular}{|c|c|c|c|c|}
\hline \multirow[t]{2}{*}{ Autori } & \multirow[t]{2}{*}{ Innovazione } & \multirow{2}{*}{$\begin{array}{l}\text { Metodo d'analisi e } \\
\text { campione d'indagine }\end{array}$} & \multicolumn{2}{|c|}{ Driver dell'innovazione } \\
\hline & & & Risorse interne & Risorse esterne \\
\hline $\begin{array}{l}\text { Avermaete et } \\
\text { al. }(2004)\end{array}$ & $\begin{array}{l}\text { Prodotto e } \\
\text { processo }\end{array}$ & $\begin{array}{l}\text { Analisi di regressione } \\
\text { Campione: } 77 \text { PMI } \\
\text { localizzate in sei regioni } \\
\text { di tre paesi europei } \\
\text { (Regno Unito, Belgio e } \\
\text { Irlanda). }\end{array}$ & $\begin{array}{l}\text { Caratteristiche } \\
\text { dell'imprenditore (età, } \\
\text { qualifica, esperienza); } \\
\text { Competenze della forza } \\
\text { lavoro (tecnici qualificati } \\
\text { e manager); } \\
\text { Investimenti in know/ } \\
\text { how (spese in attività } \\
\text { di formazione e di } \\
\text { marketing). }\end{array}$ & $\begin{array}{l}\text { Consulenze } \\
\text { (amministrative, } \\
\text { marketing e tecniche); } \\
\text { Fonti informative } \\
\text { (imprese simili, fornitori } \\
\text { di macchinari, fornitori } \\
\text { di materie prime, clienti } \\
\text { e Centri di Ricerca). }\end{array}$ \\
\hline $\begin{array}{l}\text { Batterinik et } \\
\text { al. }(2006)\end{array}$ & $\begin{array}{l}\text { Prodotto e } \\
\text { processo }\end{array}$ & $\begin{array}{l}\text { Analisi di regressione } \\
\text { Campione: } 328 \text { imprese } \\
\text { agroalimentari olandesi. }\end{array}$ & $\begin{array}{l}\text { Spese in R\&S } \\
\text { (macchinari, hardware, } \\
\text { attività di ricerche } \\
\text { interne, contrattie } \\
\text { licenze). }\end{array}$ & $\begin{array}{l}\text { Collaborazione (clienti, } \\
\text { fornitori, concorrenti, } \\
\text { Università e Centri di } \\
\text { Ricerca); } \\
\text { Fonti informative } \\
\text { (interne allazienda, } \\
\text { clienti, fornitori, } \\
\text { concorrenti, Università } \\
\text { e Centri di Ricerca); } \\
\text { Finanziamenti pubblici } \\
\text { (locali, nazionali ed } \\
\text { europei). }\end{array}$ \\
\hline $\begin{array}{l}\text { Kuhne et al. } \\
(2010)\end{array}$ & $\begin{array}{l}\text { Prodotto, } \\
\text { processo, } \\
\text { organizzative } \\
\text { marketing }\end{array}$ & $\begin{array}{l}\text { Cluster analysis } \\
\text { Campione: } 270 \text { imprese } \\
\text { coinvolte in } 90 \text { supply } \\
\text { chain (Belgio, Ungheria } \\
\text { e Italia) }\end{array}$ & $\begin{array}{l}\text { Risorse Umane (costi } \\
\text { di formazione del } \\
\text { personale, esperimenti, } \\
\text { seminari e corsi); } \\
\text { Risorse Finanziarie } \\
\text { (spese in RぬS). }\end{array}$ & $\begin{array}{l}\text { Collaborazione (clienti } \\
\text { e fornitori). }\end{array}$ \\
\hline $\begin{array}{l}\text { Karantininis } \\
\text { et al. }(2010)\end{array}$ & Prodotto & $\begin{array}{l}\text { Analisi di regressione } \\
\text { Campione: } 444 \text { imprese } \\
\text { agroalimentari danesi }\end{array}$ & $\begin{array}{l}\text { Dimensione aziendale } \\
\text { (numero di addetti). }\end{array}$ & $\begin{array}{l}\text { Integrazione verticale } \\
\text { in termini di controllo } \\
\text { gerarchico, e/o } \\
\text { contratti o altri accordi } \\
\text { di rete; } \\
\text { Potere contrattuale } \\
\text { dell'azienda rispetto } \\
\text { alle imprese a monte } \\
\text { e a valle; } \\
\text { Esportazioni (\% di } \\
\text { vendite generate dall'- } \\
\text { export). }\end{array}$ \\
\hline $\begin{array}{l}\text { Acosta et al. } \\
(2015)\end{array}$ & $\begin{array}{l}\text { Prodotto, } \\
\text { processo e } \\
\text { organizzative }\end{array}$ & $\begin{array}{l}\text { Analisi di regressione } \\
\text { Campione: } 500 \text { imprese } \\
\text { agroalimentari spagnole }\end{array}$ & $\begin{array}{l}\text { Caratteristiche } \\
\text { dell'impresa (numero } \\
\text { di addetti, \% personale } \\
\text { qualificato, spese } \\
\text { di formazione, } \\
\text { professionale, spese } \\
\text { in R\&S per addetto, } \\
\% \text { esportazioni sulle } \\
\text { vendite). }\end{array}$ & $\begin{array}{l}\text { Finanziamenti pubblici } \\
\text { (locali, nazionali ed } \\
\text { europei); } \\
\text { Fonti informative } \\
\text { (interne all'azienda, } \\
\text { fornitori, clienti, } \\
\text { concorrenti, Università, } \\
\text { fiere). }\end{array}$ \\
\hline
\end{tabular}

Fonte: nostra elaborazione 
sinergie Vol. 36, N. 105, 2018

A conclusione di questa breve rassegna, si può rilevare come la maggior parte dei lavori esaminati si siano focalizzati sulle innovazioni di prodotto e processo, considerando i comportamenti di acquisto dei consumatori, attenti alla qualità e alla sicurezza alimentare e, solitamente, molto sensibili all'utilizzo di prodotti agroalimentari con caratteristiche nuove. Un'ulteriore evidenza emersa dallanalisi della letteratura riguarda la dimensione aziendale, considerata come variabile chiave per l'innovazione. In particolare, soltanto uno dei lavori esaminati si focalizza, attraverso un'analisi field, esplicitamente sulle piccola e media dimensione (Avermaete et al., 2004), mentre gli altri studi fondano l'analisi sui dati della CIS, che utilizza come indicatori della capacità di innovazione gli investimenti in attività di R\&S, i brevetti e il numero di nuovi prodotti realizzati. Va detto, tuttavia, che le PMI possono innovare anche senza svolgere attività di R\&S internamente, attraverso lo sviluppo di collaborazioni esterne. La rilevanza della dimensione e della collaborazione ha contribuito, inoltre, ad alimentare il tema delle politiche di sostegno allinnovazione, come sottolineato da Acosta et al. (2015) che evidenziano la necessità di definire azioni di policy specifiche per le PMI agroalimentari.

\section{Il framework di analisi della capacità di innovazione}

Sulla base della letteratura analizzata nel paragrafo precedente, i lavori di Acosta et al. (2015), Avermaete et al. (2004) e Kuhne et al. (2010) rappresentano i riferimenti più appropriati per analizzare un contesto poco esplorato, quale quello della delle filiere agroalimentari in Campania, caratterizzato principalmente da micro e piccole imprese. Il framework proposto considera l'influenza di alcune risorse, interne ed esterne allimpresa, sulla capacità di innovazione delle PMI e delle microimprese agroalimentari (Figura 1).

Le risorse interne fanno riferimento alla struttura ed alle caratteristiche dellimpresa, ed in particolare: la dimensione aziendale (in termini di classe di addetti e di fatturato), il personale qualificato, le esportazioni e gli investimenti in R\&S. La dimensione aziendale e gli investimenti in R\&S rappresentano le variabili chiave analizzate in tutti gli studi citati, e le indagine realizzate confermano lesistenza di un legame tra dimensione ed investimenti in R\&S. Con riferimento alla piccola e media dimensione il contributo sia delle risorse umane che quelle finanziare sulla capacità di innovazione richiede una specifica analisi, che ad oggi gli studi nel settore agroalimentare non hanno ancora offerto.

Le esportazioni rappresentano unaltra variabile che influisce sulla capacità innovativa delle imprese, in quanto la competizione sui mercati internazionali incentiva le imprese ad attivare nuovi processi innovativi (Karantininis et al., 2010). Lapertura ai mercati esteri offre la possibilità di conoscere le innovazioni intraprese dai competitors e, quindi, attivare un processo di innovazione anche nel mercato locale. In linea con il lavoro di Avermaete et al. (2004), è stata considerata come ulteriore risorsa interna la presenza di personale qualificato, che contribuisce alla realizzazione di innovazioni attraverso la realizzazione di nuove tecniche volte al 
miglioramento del processo produttivo o alla realizzazione di nuovi prodotti in-house.

Marcella De Martino Fabio Magnotti Lodovico Santoro

Poiché la piccola e piccolissima dimensione aziendale presentano Linnovazione nelle vincoli in termini di risorse finanziare e manageriali interne, risulta $\begin{gathered}\text { agroalimentari della } \\ \text { Regione Campania }\end{gathered}$ piccole e medie imprese necessario estendere l'analisi alle risorse esterne a cui l'azienda può accedere. Ladozione di un approccio di "innovazione aperta" per lo sviluppo di innovazioni è ormai indispensabile nell'attuale scenario competitivo, in cui gli elevati costi legati all'innovazione e la necessità di conoscenze e competenze specifiche impediscono a molte imprese, soprattutto quelle di piccole e medie dimensioni, di svolgere attività innovative e di R\&S. Il raggiungimento di un vantaggio competitivo è, soprattutto in alcuni settori fortemente concorrenziali come quello dell'agroalimentare, subordinato a quello che Kanter (1994) definisce vantaggio collaborativo. Ed è proprio sulla base di tali considerazioni, che si è scelto di misurare, oltre che i finanziamenti pubblici e le fonti informative, anche la collaborazione esterna. In particolare, sebbene rilevanti i rapporti con fornitori e clienti, l'analisi si estende ai rapporti di collaborazione con altri soggetti detentori di conoscenza come Università, istituti di ricerca, associazioni professionali e consulenti. Le fonti informative, ovvero i principali canali di informazione utile ai fini dell'innovazione, dipendono dalla tipologia dei soggetti con cui l'impresa interagisce quali: fornitori, consumatori, competitors, consulenti, Università, associazioni professionali, fiere e convegni, nonché personale interno all'azienda (Avermaete et al., 2004). Infine, i finanziamenti pubblici rappresentano una variabile rilevante per migliorare la capacità innovativa delle PMI, in quanto accedendo a fondi locali e/o nazionali, le imprese hanno una maggiore propensione ad investire in attività di R\&S (Acosta et al., 2015).

Fig. 1: Il framework di analisi della capacità innovativa delle PMI agroalimentari

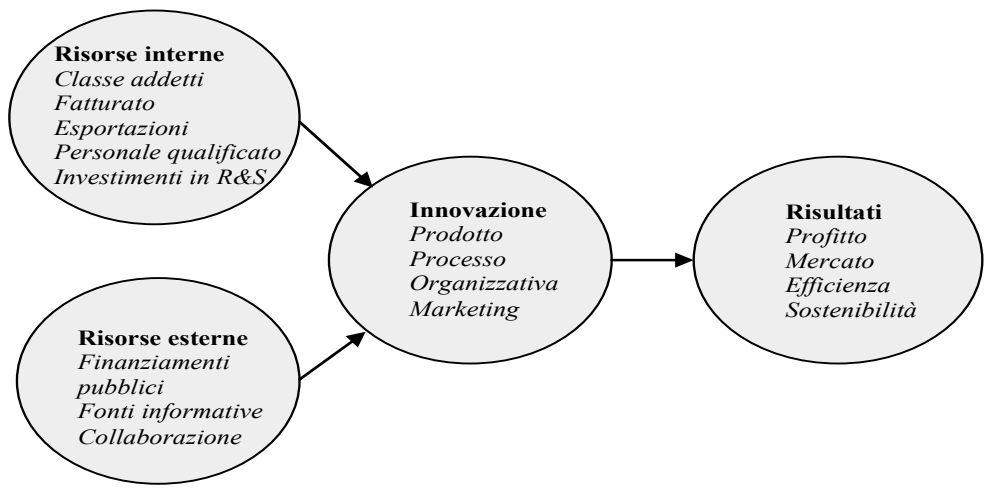

Fonte: nostra elaborazione

Poiché i cambiamenti adottati dalle imprese agroalimentari sono spesso incrementali e riguardano non solo il processo produttivo e la qualità dei prodotti realizzati, ma anche la riorganizzazione delle attività aziendali e delle attività di marketing, il framework analizza quattro tipologie di innovazione: prodotto, processo, organizzative e di marketing. 
sinergie Vol. 36, N. 105, 2018

Infine, il framework considera l'impatto che l'innovazione può generare sia direttamente sui risultati aziendali, come ad esempio la crescita della quota di mercato e del profitto, sia in maniera indiretta sugli altri stakeholders, come ad esempio la sostenibilità ambientale.

In linea con gli studi citati, lassenza di tali fattori possono rappresentare i principali ostacoli all'innovazione per le imprese e sono stati considerati nellanalisi svolta presso le PMI agroalimentari della Campania. In particolare, tali fattori inabilitanti possono afferire alla sfera interna dell'impresa, come la mancanza di risorse finanziarie da poter investire in attività di R\&S, l'impossibilità di poter sopportare gli alti costi dell'innovazione, l'assenza di competenze (Avermaete et al., 2004); oppure dipendere dal contesto in cui l'impresa opera, come l'assenza di informazioni sui progressi scientifici e tecnologici, la difficoltà a trovare partner con cui collaborare (Kuhne et al., 2010) o nel poter accedere alle fonti di finanziamento esterne (Acosta et al., 2015).

Struttura del questionario, variabili utilizzate e relativa scala di misurazione

Coerentemente con il framework teorico, il questionario è stato strutturato in modo da analizzare le quattro dimensioni del framework d'analisi: risorse interne ed esterne, innovazioni e risultati. In relazione alle risorse interne, la dimensione aziendale viene rilevata attraverso classi di fatturato e numero di addetti. Al fine di tenere conto della diffusa presenza delle micro imprese nel tessuto imprenditoriale della Campania, il fatturato è stato suddiviso in classi più ridotte rispetto a quelle impiegate dalle statistiche ufficiali ${ }^{4}$. Con riferimento al numero di addetti, è stata invece utilizzata la classificazione delle $\mathrm{PMI}^{5}$ proposta dall'Unione Europea (European Commission, 2003). Le esportazioni, misurate dalla percentuale di produzione assorbita dai mercati esteri, sono state raggruppate in quattro classi di ampiezza nulla, bassa, media o alta $^{6}$. La variabile "personale qualificato" è stata rilevata attraverso una scelta dicotomica (si/no) tra differenti figure professionali presenti nelle imprese agroalimentari: tecnologi alimentari, medici dietologi, figure tecniche non laureate, chimici, ingegneri ed altro ${ }^{7}$. Gli investimenti in R\&S sono stati misurati considerando la percentuale del fatturato investito in attività di $R \& S^{8}$.

$4 \quad$ Le seguenti classi di fatturato sono state discusse e validate dalle Associazioni di categoria: fino a $€ 50.000$, da $€ 50.000$ a $€ 250.000$; da $€ 250.000$ a 1 milione di euro; da 1 milione di euro a 2 milioni di euro; oltre i 2 milioni di euro.

5 La categoria delle piccole e medie imprese (PMI) è costituita da imprese che occupano meno di 250 persone. All'interno della categoria delle PMI, si definisce piccola impresa un'impresa che occupa meno di 50 persone, mentre si definisce micro impresa un'impresa con meno di 10 persone.

6 Le seguenti classi di esportazione sono state discusse e validate dalle Associazioni di categoria: nulla, bassa (meno del $30 \%$ ); media (tra il 30\% e 60\%); alta (oltre il 60\%).

7 La scelta delle figure professionali attinenti alla variabile personale qualificato sono state discusse e validate dalle Associazioni di categoria.

8 Le seguenti classi di investimenti in R\&S sono state discusse e validate dalle Associazioni di categoria: nessuna, meno dell'1\%, tra l'1-2\%, tra il 2-4\% e maggiore del $4 \%$. 
Con riferimento alle seconda dimensione del framework relativa alle risorse esterne, il ricorso ai finanziamenti pubblici è stato rilevato attraverso una scelta dicotomica (si/no) a seconda che l'azienda ne fruisca o meno. Nel caso affermativo, è stata utilizzata una risposta a scelta multipla per esplorare sia il contesto geografico (europeo, nazionale e regionale) che le diverse tipologie di finanziamento (Programmi Quadro, bandi del Ministero delle Attività Produttive, Piano di Sviluppo Rurale, Programma Operativo Regionale e altre fonti). Le fonti informative sono state analizzate attraverso una scelta multipla tra differenti tipologie di fonti: interne (all'interno dell'azienda o del gruppo), di mercato (fornitori di materie prime, macchinari, o software, competitors e clienti), istituzionali (Università, istituti di ricerca ed istituzioni pubbliche) ed altre (convegni, fiere e associazioni professionali). La collaborazione, infine, è misurata attraverso una scelta multipla tra i vari tipi di partner (fornitori di materie prime, fornitori di macchinari e software, clienti, competitors, consulenti, Università ed istituti di ricerca, e associazioni professionali) e la loro posizione geografica (Italia, Europa, Stati Uniti ed altro).

La terza dimensione del framework analizza le quattro tipologie di innovazione (prodotto, processo, organizzative e di marketing) sia attraverso scelte dicotomiche (si/no) in merito alla presenza o meno delle attività indicate, sia attraverso risposte aperte che hanno prodotto informazioni di tipo qualitativo. In particolare, dopo aver fornito agli intervistati le definizioni riguardanti le diverse tipologie di innovazione, è stato rilevato il grado di innovatività (incrementale e radicale) attraverso una descrizione dettagliata delle innovazioni adottate negli ultimi tre anni.

Nella quarta dimensione del framework, sono analizzati i risultati derivanti da ciascuna tipologia di innovazione, attraverso un insieme di indicatori di performance riguardanti: il profitto (trend crescente); il mercato (ingresso in un nuovo mercato, aumento della quota di mercato); l'efficienza (miglioramento della flessibilità e della capacità produttiva, riduzione dei costi di produzione); la sostenibilità (aumento della qualità dell'ambiente lavorativo, riduzione dell'impatto ambientale, soddisfazione della normativa).

Infine, una specifica sezione del questionario è volta a rilevare la percezione delle imprese riguardo ai principali fattori di ostacolo ai processi innovativi. Tali ostacoli sono rappresentati da fattori economici (quali la mancanza di risorse interne, di fonti di finanziamento esterne e costi per l'innovazione troppo elevati); dalla difficoltà dell'impresa di assorbire nuove tecnologie o nuove conoscenze (a causa della mancanza di personale qualificato, della scarsa conoscenza delle tecnologie disponibili e difficoltà nel trovare partner nello sviluppo di innovazioni); dalle condizioni avverse di mercato (mercato dominato da imprese leader; incertezza della domanda).

La struttura del questionario, la scelta delle variabili e delle relative scale di misurazione sono state discusse e convalidate dalle Associazioni di Categoria (Coldiretti, Unione Industriali Napoli). 
$\underset{\text { italian journal of management }}{\text { Sinergie }}$

Vol. 36, N. 105, 2018

\section{L'indagine empirica}

\subsection{Il Campione d'indagine e metodo di analisi dei dati}

L'universo di riferimento delle imprese è stato definito utilizzando lo studio condotto da De Martino et al. (2014) sull'analisi delle filiere agroalimentari in Campania. Rispetto ai dati Istat, i risultati di tale analisi consentono sia di mappare la distribuzione delle imprese agroalimentari campane nell'ambito dei singoli stadi produttivi, sia di delineare le relazioni di subfornitura e i rapporti proprietari tra tali imprese. Secondo questo studio, sul territorio campano erano localizzate 620 imprese di produzione dei derivati del latte, 308 imprese di produzione di oli e grassi vegetali, 442 imprese di paste alimentari, cuscus e prodotti farinacei e 360 imprese produttrici di vino. Per reperire i contatti aziendali si è utilizzata la Banca dati Aida e quella dell'Osservatorio della Cooperazione Agricola italiana raccogliendo informazioni per contattare 453 imprese.

Poiché l'invio del questionario via e-mail si è dimostrato inefficace, si è deciso di coinvolgere alcune associazioni di categoria (Coldiretti e Unione Industriale di Napoli) nella fase di selezione e somministrazione del questionario. In linea con il framework proposto, ai fini della selezione delle imprese da coinvolgere nell'indagine sono stati adottati i seguenti criteri:

- dimensione aziendale (piccola e media);

- posizionamento a valle della filiera di riferimento e presenza di un marchio proprio;

- esportazione dei prodotti all'estero.

La scelta di selezionare le piccole e medie imprese della regione Campania scaturisce, chiaramente, dalla necessità di esplorare le dinamiche che caratterizzano i processi innovativi di realtà produttive così diffuse sul territorio italiano e campano, ma che sfuggono alle statistiche ufficiali, sia europee che nazionali. Inoltre, al fine di individuare PMI innovative è stato necessario selezionare imprese posizionate a valle della filiera di riferimento. Queste ultime, infatti, avendo una maggior conoscenza delle esigenze del consumatore finale, driver fondamentale per l'innovazione, hanno una maggiore propensione ad innovare rispetto a fornitori e distributori. Un ulteriore criterio nella selezione del campione è rappresentato dalla propensione alle esportazioni. Tuttavia, quest'ultimo criterio è stato tenuto parzialmente in considerazione poiché solo una percentuale molto esigua di imprese di piccola dimensione esporta i propri prodotti allestero. Considerando soltanto le imprese esportatrici, oltre ad avere una ridotta numerosità campionaria, non sarebbe stato possibile catturare i diversi fattori che influenzano la capacità innovativa delle piccole imprese agroalimentari.

Delle 453 imprese contattate, soltanto 122 si sono dichiarate disposte a partecipare all'indagine empirica attraverso la compilazione di un questionario presso la sede aziendale. Le interviste sono state condotte tra settembre 2014 e febbraio 2015 da due intervistatori appositamente formati per assistere i proprietari e/o i manager delle imprese nella compilazione del questionario. 
La distribuzione per filiera delle imprese del campione finale è riportata in Tabella 2. Le filiere lattiero-caseario e olivicola sono rappresentative dell'universo di riferimento mentre la bassa presenza di imprese appartenenti alla filiera cerealicola-pastaria è dovuta principalmente a fattori strutturali del comparto campano. Tale filiera risulta infatti dominata da imprese di grandi dimensioni che non rispecchiano il primo criterio di selezione del campione. Inoltre, alcune delle imprese cerealicolepastarie di minori dimensioni, operando prevalentemente in qualità di subfornitori, non sono state incluse, in quanto non rispettavano il secondo criterio di selezione.

Tuttavia, la rappresentatività del campione è stata testata attraverso il test di ipotesi sulla differenza tra due medie. In tal senso, è stata utilizzata come variabile il numero medio dei dipendenti per impresa sia per il campione che per l'universo di riferimento e per le diverse filiere.

Utilizzando la statistica t-Student, il campione risultava rappresentativo dell'universo di riferimento con un grado di significatività dell' $1 \%{ }^{9}$. Tale risultato conferma in tal modo la rappresentatività del campione ottenuto, anche considerando la ripartizione delle imprese tra le quattro filiere considerate.

Tab. 2: Struttura delluniverso di riferimento e del campione

\begin{tabular}{|l|c|c|c|c|}
\hline \multirow{2}{*}{ Filiera } & \multicolumn{2}{|c|}{ Universo di riferimento } & \multicolumn{2}{c|}{ Campione } \\
\cline { 2 - 5 } & $\begin{array}{c}\text { Numero } \\
\text { di imprese }\end{array}$ & $\begin{array}{c}\text { Incidenza \% } \\
\text { della filiera }\end{array}$ & $\begin{array}{c}\text { Numero } \\
\text { di imprese }\end{array}$ & $\begin{array}{c}\text { Rappresentatività } \\
\%\end{array}$ \\
\hline Lattiero-Casearia & 620 & 35,8 & 43 & 6.9 \\
\hline Vitivinicola & 360 & 20,8 & 47 & 13.1 \\
\hline Olivicola & 308 & 17,8 & 18 & 5.8 \\
\hline Cerealicola-pastaria & 442 & 25,6 & 14 & 3.1 \\
\hline Totale & 1730 & & 122 & \\
\hline
\end{tabular}

Fonte: nostra elaborazione

I dati raccolti attraverso i 122 questionari sono stati elaborati utilizzando la Two-step Cluster analysis. Tale tecnica, sviluppata da Chiu et al. (2001), consente di raggruppare le imprese in un numero di cluster caratterizzati dalla massima omogeneità interna ai gruppi e la massima eterogeneità tra i gruppi, rispetto a determinati fattori, rappresentati dalle variabili di clustering. Tali fattori dovrebbero fornire una chiara differenziazione dei differenti gruppi rispetto ad un dato obiettivo conoscitivo e la loro scelta rappresenta una fase cruciale in ogni Cluster analysis. A tal fine, le variabili di clustering sono state inizialmente individuate tra quelle che presentano una correlazione inferiore a 0,7 rispetto alle altre come suggerito da Mooi e Sarstedt (2011). Inoltre, tali variabili devono offrire il maggiore potere

9 Essendo la varianza della popolazione ignota, il test di significatività della media è stato condotto utilizzando la statistica t-Student anziché la Normale. Il valore empirico della $t$-Student $(0,65)$ ricadeva nella regione di accettazione delimitata dai valori teorici $( \pm 1,982)$. Pertanto veniva accettata l'ipotesi nulla, secondo la quale la media del campione non era significativamente diversa dalla media della popolazione, fino ad un livello di significatività pari all' $1 \%$. 
sinergie Vol. 36, N. 105, 2018

predittivo del fenomeno indagato. Pertanto, le variabili di clustering così scelte risultano essere la "dimensione aziendale", la "classe di addetti", il "fatturato", le "esportazioni", gli "investimenti in R\&S”, i "finanziamenti pubblici" e la "collaborazione" (De Martino e Magnotti, 2018). Rispetto ad altre tecniche di cluster, come la k-means o la cluster gerarchica, la procedura two-step ha il vantaggio sia di definire automaticamente il numero ottimale di cluster, evitando una scelta arbitraria e predeterminata, sia di analizzare variabili continue e categoriche. Infine, attraverso la procedura two-step è possibile stimare l'importanza di ciascun predittore, ovvero il contributo di ogni variabile di clustering nella segmentazione del campione. Nella presente analisi, le variabili con il maggior potere predittivo risultavano gli "investimenti in R\&S”, la "classe di addetti" e il "fatturato", mentre le "esportazioni”, i "finanziamenti pubblici" e la "collaborazione" presentavano un punteggio di importanza inferiore a 0,5 su una scala da 0 a 1 . Tale risultato consente di individuare una connessione tra alcune variabili e l'appartenenza ai cluster.

\subsection{L'innovazione delle PMI agroalimentari della Campania}

Lelevato grado di dettaglio delle informazioni raccolte rappresenta un aspetto particolarmente importante del presente lavoro in quanto, diversamente dalle statistiche disponibili sia a livello europeo che nazionale, consente di analizzare congiuntamente fattori legati all'innovazione, alle caratteristiche aziendali e al contesto di riferimento. Va precisato che cinque delle imprese selezionate nel campione sono state escluse dall'analisi a causa dell'assenza di informazioni relative ai dati anagrafici e alle variabili di clustering. I risultati finali, pertanto, fanno riferimento ai dati rilevati su 117 imprese.

Un primo risultato, riguarda la distribuzione del campione tra imprese innovative e non innovative. La Tabella 3 evidenzia che 51 imprese (pari al $43,5 \%$ ) hanno realizzato almeno una innovazione, mentre le rimanenti 66 non ne hanno introdotto per motivi legati sia a fattori interni all'azienda che a fattori di contesto. Considerando il dato disaggregato per singola filiera, emerge poi che la filiera vitivinicola comprende la più alta percentuale di imprese che hanno innovato nel periodo preso in esame (il 52,9\% sul totale delle imprese innovative), mentre in quella olivicola si registra la percentuale più bassa $(5,9 \%)$.

Tab. 3: L'innovazione nelle singole filiere

\begin{tabular}{|l|c|c|}
\hline & \multicolumn{1}{|c|}{$\begin{array}{c}\text { Imprese innovative } \\
\text { Numero (\%) }\end{array}$} & $\begin{array}{c}\text { Imprese non innovative } \\
\text { Numero (\%) }\end{array}$ \\
\hline Filiera agroalimentare & & $25(37,9)$ \\
\hline Lattiero - Casearia & $15(29,4)$ & $19(28,8)$ \\
\hline Vitivinicola & $27(52,9)$ & $15(22,7)$ \\
\hline Olivicola & $3(5,9)$ & $7(10,6)$ \\
\hline Cerealicola-pastaria & $6(11,8)$ & $66(56,5)$ \\
\hline$N(\%)$ & $51(43,5)$ & \\
\hline
\end{tabular}

Fonte: nostra elaborazione 
L'applicazione della cluster analysis, ha permesso, poi, di identificare tre gruppi di imprese caratterizzati da altrettanti innovation modes, definiti rispettivamente: Innovative e Collaborative (IC), Innovative e Non Collaborative (INC), Non Innovative (NI) (Tabella 4).

Tab. 4: Gli innovation modes delle PMI agroalimentari (frequenze \%)

\begin{tabular}{|c|c|c|c|c|c|c|c|c|}
\hline & \multirow{2}{*}{$\begin{array}{c}\text { Driver } \\
\text { dell'innovazione }\end{array}$} & \multirow{2}{*}{$\begin{array}{l}\text { Cluster } 1 \\
\text { IC }\end{array}$} & \multirow{2}{*}{$\begin{array}{l}\text { Cluster } 2 \\
\text { INC }\end{array}$} & \multicolumn{2}{|c|}{$\begin{array}{l}\text { Cluster } 3 \\
\text { NI }\end{array}$} & \multirow{2}{*}{$\begin{array}{c}\text { Totale } \\
\text { Campione }\end{array}$} & \multirow{2}{*}{ ANOVA } & \multirow[t]{2}{*}{ Sign. } \\
\hline & & & & MNI & PMINI & & & \\
\hline \multirow{22}{*}{ 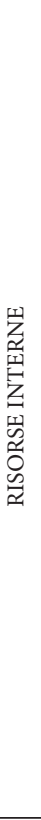 } & $N .^{\circ}$ Addetti & & & & & & 48,402 & 000 \\
\hline & Micro (1-9) & 5,3 & 62,5 & 100 & 4,0 & 53,8 & & \\
\hline & Piccole (10-49) & 84,2 & 37,5 & - & 88,0 & 42,8 & & \\
\hline & Medie (50-249) & 10,5 & - & - & 8,0 & 3,4 & & \\
\hline & Fatturato & & & & & & 53,273 & 000 \\
\hline & $€ 0-50.000$ & - & 6,3 & 43,9 & - & 17,1 & & \\
\hline & $€ 50.000-250.000$ & 5,3 & 68,8 & 56,1 & 16,0 & 42,7 & & \\
\hline & $€ 250.000$ - 1 milione & 42,1 & 6,3 & - & 40,0 & 17,1 & & \\
\hline & $€ 1-2$ milione & 21,1 & 18,8 & - & 36,0 & 16,2 & & \\
\hline & $€ 2$ milione ed oltre & 31,6 & - & - & 8,0 & 6,8 & & \\
\hline & Investimenti in R\&S & & & & & & 113,694 & 000 \\
\hline & Nessuna & - & 3,1 & 100 & 100 & 57,2 & & \\
\hline & Meno dell'1\% & 31,6 & 12,5 & - & - & 8,5 & & \\
\hline & Tra l'1-2\% & 5,3 & 71,9 & - & - & 20,5 & & \\
\hline & Tra il 2-4\% & 36,8 & 9,4 & - & - & 8,5 & & \\
\hline & Maggiore del 4\% & 26,3 & 3,1 & - & - & 5,1 & & \\
\hline & Personale Qualificato & & & & & & & \\
\hline & No & 31,6 & 37,9 & 100 & 100 & 72,8 & & \\
\hline & $\mathrm{Si}$ & 68,4 & 62,1 & - & - & 27,2 & & \\
\hline & Quota esportazioni & & & & & & 4,054 & ,009 \\
\hline & No & 26,3 & 40,6 & 100 & 100 & 71,8 & & \\
\hline & $\mathrm{Si}$ & 73,7 & 59,4 & - & - & 28,2 & & \\
\hline \multirow{7}{*}{ 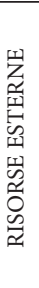 } & Collaborazione & & & & & & 18,359 & 000 \\
\hline & No & 47,4 & 81,2 & 100 & 100 & 86,3 & & \\
\hline & $\mathrm{Si}$ & 52,6 & 18,8 & - & - & 13,7 & & \\
\hline & Fonti Informative & & & & & & & \\
\hline & No & & 18,8 & 100 & 100 & 61,5 & & \\
\hline & $\mathrm{Si}$ & 100 & 81,2 & - & - & 38,5 & & \\
\hline & $\begin{array}{l}\text { Dimensione cluster, } N \\
(\%)\end{array}$ & $\begin{array}{c}19 \\
(16,2)\end{array}$ & $\begin{array}{c}32 \\
(27,3)\end{array}$ & $\begin{array}{c}41 \\
(35,1)\end{array}$ & $\begin{array}{c}25 \\
(21,4)\end{array}$ & $\begin{array}{c}117 \\
(100)\end{array}$ & & \\
\hline
\end{tabular}

Fonte: nostra elaborazione

In base alla dimensione aziendale, le imprese non innovative sono poi state suddivise in Microimprese Non Innovative (MNI) e Piccole e Medie Imprese Non Innovative (PMINI). Nelle ultime due colonne della Tabella 4 sono riportati i risultati dell'Analysis of Variance (ANOVA) e la loro significatività statistica limitatamente alle variabili di clustering. La colonna ANOVA indica in che misura tali variabili hanno contributo alla individuazione dei cluster. Le variabili che hanno contribuito maggiormente alla segmentazione del campione risultano essere investimenti in R\&S e la dimensione aziendale (sia per fatturato che classe di addetti) con un alto livello di significatività. 


\section{sinergie}

Vol. 36, N. 105, 2018

\subsection{La Cluster analysis}

\subsubsection{Le imprese Innovative e Collaborative (IC)}

Tale gruppo rappresenta il 16,2\% del campione (Tabella 4) ed è composto per l'84,2\% da piccole imprese (10-49 dipendenti) e per il 10,5\% da imprese di medie dimensioni (50-249 addetti). Tali imprese appartengono principalmente alla filiera vitivinicola $(36,8 \%)$, lattiero-casearia $(31,6 \%)$ e cerealicola-pastaria $(26,3)$. La maggior parte delle IC presenta un fatturato annuo superiore ad un milione di euro e tutte impiegano una quota del proprio fatturato in investimenti in R\&S.

Nel $73,7 \%$ dei casi, le imprese in questione riescono ad accedere ai finanziamenti pubblici soprattutto attraverso la partecipazione al Programma Operativo Regionale (POR) della Regione Campania. Da una analisi di approfondimento emerge che il Piano di Sviluppo Rurale (PSR) della Campania rappresenta una fonte di finanziamento strategica per rafforzare ed incentivare i processi innovati nella regione. Al contrario, i programmi europei (VII Programma Quadro 2007-2013) hanno un peso marginale per le imprese in esame, in quanto solo due di esse hanno dichiarato di aver partecipato a progetti europei. La maggiore incidenza di finanziamenti provenienti da istituzioni localizzate sul territorio conferma la rilevanza della prossimità nelle scelta di innovazione da parte delle PMI. Tale tendenza è legata al vantaggio che la prossimità fisica genera per le imprese sia nello scambio di informazioni che nello sviluppare relazioni dirette con differenti soggetti chiave del contesto locale.

Una peculiarità che contraddistingue questo cluster è la capacità di sviluppare collaborazioni per la realizzazione di innovazioni $(52,6 \%)$. Come si evince dalla Tabella 5, le imprese Innovative e Collaborative hanno attivato rapporti di collaborazione principalmente con interlocutori o controparti nazionali e, segnatamente: all'interno dell'azienda o del Gruppo di appartenenza $(31,2 \%)$, con i consulenti $(31,2 \%)$, con Università ed Istituti di ricerca pubblici $(31,2 \%)$, con i clienti $(18,7 \%)$ e con le associazioni professionali (12,5\%). Gli accordi siglati con i competitors, invece, hanno principalmente riguardato partner europei (6,7\%), nell'ambito dei due casi di progetti finanziati dai Programmi Quadro dell’Unione Europea.

Tab. 5: I partner più rilevanti per le attività di innovazione (\% risposta multipla)

\begin{tabular}{|c|c|c|c|}
\hline & $\begin{array}{l}\text { Innovative e } \\
\text { Collaborative }\end{array}$ & $\begin{array}{c}\text { Settore } \\
\text { Manifatturiero } \\
\text { Italia }^{*}\end{array}$ & $\begin{array}{c}\text { Settore } \\
\text { Agroalimentare } \\
\text { Italia* }^{*}\end{array}$ \\
\hline All'interno dell'azienda & $31,2 \%$ & $4,0 \%$ & $0,8 \%$ \\
\hline Fornitori di materie prime & $31,3 \%$ & \multirow{2}{*}{$10,1 \%$} & \multirow{2}{*}{$3,6 \%$} \\
\hline Fornitori di macchinari, software & $43,7 \%$ & & \\
\hline Clienti e consumatori & $18,7 \%$ & $3,9 \%$ & $2,7 \%$ \\
\hline Competitors od altre aziende del settore & $6,7 \%$ & $5,1 \%$ & $3,3 \%$ \\
\hline Consulenti & $31,2 \%$ & $9,0 \%$ & $9,1 \%$ \\
\hline Università ed istituti di ricerca pubblici & $31,2 \%$ & $7,0 \%$ & $8,5 \%$ \\
\hline Associazioni professionali o industriali & $12,5 \%$ & N.D. & N.D. \\
\hline
\end{tabular}

Fonte: Nostra elaborazione; * Eurostat - Community Innovation Survey 2014 
Una rilevanza non trascurabile per il successo del processo innovativo è stata poi attribuita ai fornitori di materie prime $(31,3 \%)$ e di macchinari e software (43,7\%). Confrontando i risultati della presente ricerca con quelli dell'indagine CIS 2014, si può constatare come le forme di partnership siano nettamente superiori sia rispetto al settore manifatturiero che a quello agroalimentare. In particolare, emerge come il contesto campano, fotografato dalla nostra indagine, sia caratterizzato da una maggiore propensione ad innovare lungo la supply chain rispetto al contesto italiano. Tale tendenza è probabilmente legata alla numerosa presenza di microimprese che, in assenza di risorse, avviano processi innovativi con soggetti conosciuti e con cui hanno già rapporti di fiducia pregressi, come i fornitori di materie prime. Anche i fornitori di macchinari e software sono soggetti con cui l'impresa collabora, mostrando una predilezione delle piccole imprese a fondare l'attività innovativa sull'acquisizione di tecnologie incorporate in macchinari e d impianti innovativi. Inoltre, osservando i dati relativi alle collaborazioni con Università e istituti di ricerca pubblici, va rilevato come il sistema della ricerca in Campania, a differenza del contesto nazionale, rappresenti per le PMI un partner fondamentale per attingere a conoscenze scientifiche e tecnologiche difficilmente accessibili.

Tra le fonti informative più rilevanti, le imprese hanno poi indicato le fiere come il luogo in cui hanno avuto modo di incontrare le professionalità e le competenze più significative per implementare i progetti di innovazione, ma anche unopportunità di scambio di conoscenze e know-how.

Rilevante ai fini della realizzazione del processo innovativo, sono risultate altresì le competenze presenti in azienda e, in particolare, quelle in possesso di tecnologi alimentari, ingegneri e chimici.

Il forte orientamento alle esportazioni che caratterizza le imprese di questo cluster consente un più ampio accesso alle conoscenze scientifiche disponibili nei diversi mercati di sbocco; di alimentare il processo innovativo con idee nuove; di poter imitare ed integrare nei propri prodotti e processi produttivi le conoscenze acquisite da altre imprese.

Con specifico riferimento alle tipologie di innovazione realizzate, le IC si sono concentrate prioritariamente sulle innovazioni di prodotto $(89,5 \%)$ e di processo $(68,4 \%)$ (Tabella 6). Sebbene in misura meno pronunciata, si registra anche l'adozione di innovazioni nel marketing $(47,4 \%)$ e nel miglioramento dei sistemi gestionali e organizzativi $(21,1 \%)$.

In particolare, dalle informazioni aggiuntive fornite durante le interviste, le innovazioni di prodotto sono state indirizzate prevalentemente a soddisfare le nuove esigenze dei consumatori, come ad esempio i vini a basso tenore alcolico, i vini vegani o i formaggi con caglio vegetale. Inoltre, sono state introdotte anche innovazioni tese a migliorare la shelf life dei prodotti, come nel caso della realizzazione di una nuova tecnica di congelamento della mozzarella di bufala per le fasi del trasporto. Per quanto concerne invece le innovazioni di processo, estremamente rilevante è il ruolo svolto dall'uso di macchinari altamente tecnologici che hanno portato, ad esempio, all'adozione del Voluntary Milking System (VMS), un sistema di mungitura automatico tramite chip, o all'introduzione di nuovi strumenti per la crio-macerazione delle uve che consente una fermentazione a freddo dei mosti. La realizzazione di 
sinergie

italian journal of management Vol. 36, N. 105, 2018

tali processi è il risultato della collaborazione delle imprese con fornitori altamente specializzati o con istituti di ricerca ed Università.

Tab. 6: Tipologie di innovazione nelle imprese dei primi due cluster

\begin{tabular}{|l|c|c|c|}
\hline & IC (\%) & INC (\%) & Campione (\%) \\
\hline Innovazione di prodotto & & & \\
\hline $\mathrm{No}$ & 10,5 & 31,2 & 66,7 \\
\hline $\mathrm{Si}$ & 89,5 & 68,8 & 33,3 \\
\hline Innovazione di processo & & & \\
\hline $\mathrm{No}$ & 31,6 & 50,0 & 75,2 \\
\hline $\mathrm{Si}$ & 68,4 & 50,0 & 24,8 \\
\hline Innovazioni organizzative & & & \\
\hline $\mathrm{No}$ & 78,9 & 93,7 & 94,9 \\
\hline $\mathrm{Si}$ & 21,1 & 6,3 & 5,1 \\
\hline Innovazione di marketing & & & \\
\hline $\mathrm{No}$ & 52,6 & 56,2 & 80,3 \\
\hline $\mathrm{Si}$ & 47,4 & 43,8 & 19,7 \\
\hline $\begin{array}{l}\text { Dimensione dei cluster, } \\
\text { N (\%) }\end{array}$ & $19(16,2)$ & $32(27,3)$ & $117(100)$ \\
\hline
\end{tabular}

Fonte: nostra elaborazione

Si registra, invece, una incidenza relativamente bassa di innovazioni organizzative $(21,1 \%)$ tese principalmente a migliorare i sistemi di gestione delle conoscenze o lo scambio di informazioni internamente allimpresa. Tale risultato è connesso alla piccola dimensione aziendale e a una gestione a conduzione prevalentemente familiare che non richiede ladozione di sistemi informativi e/o modelli gestionali interni. Più significative sono, invece, le innovazioni avviate nell'area del marketing $(47,4 \%)$, che consentono di realizzare cambiamenti significativi nellattività di vendita o di distribuzione, come ad esempio nel caso della realizzazione di piattaforme di e-commerce.

\subsubsection{Le imprese Innovative e Non Collaborative (INC)}

Questo gruppo rappresenta il $27,3 \%$ del campione ed è formato per il $62,5 \%$ da microimprese (1-9 addetti) e per il restante $37,5 \%$ da imprese di piccole dimensioni, con meno di 49 addetti (Tabella 4). La limitata dimensione è confermata dal modesto livello del fatturato che nella maggior parte dei casi è inferiore a €250.000. Questa caratteristica influisce, ovviamente, anche sul livello degli investimenti in innovazione (R\&S) che per la maggior parte del cluster risulta inferiore al $2 \%$ del fatturato. Si tratta, comunque, di un dato che denota una certa propensione all'innovazione di queste imprese che, anche in questo caso, si concentrano principalmente nelle filiera vitivinicola $(62,5 \%)$ e in quella lattierocasearia $(28,1 \%)$. Considerando le risorse interne, risulta che nel $62,1 \%$ dei casi, nei processi innovativi viene impiegato personale qualificato, in particolare tecnici non laureati e tecnologi alimentari. Le risorse esterne maggiormente utilizzate sono, invece, le fonti informative $(81,2 \%)$ ed il ricorso a finanziamenti esterni $(59,4 \%)$. Inoltre, risultano ancora limitate 
le imprese che utilizzano forme di collaborazione esterna $(18,8 \%)$ al fine di avviare attività innovative.

Da domande di approfondimento durante le interviste emerge che nella fase di avvio del processo di innovazione, le imprese ricercano notizie di vario genere. Nella maggior parte dei casi queste riguardano i progressi tecnologici e scientifici e le relative ricadute sullefficienza del processo produttivo. Tali notizie vengono spesso ricercate presso molteplici fonti di informazione, anche molto diverse tra di loro, dalle quali desumere le modalità con cui si evolve l'innovazione all'interno del settore o lungo la filiera di appartenenza. A tal proposito, dalla maggior parte delle interviste emerge che le informazioni più importanti per l'avvio del processo di innovazione sono quelle che provengono dall'interno dell'azienda. Sono indicati inoltre come fonti di acquisizione di conoscenza i convegni e le fiere, i clienti e le Associazioni professionali. I fornitori di materie prime, a differenza di quanto è emerso per le imprese del cluster IC, non risultano condizionare il processo innovativo delle INC, dal momento che in molte filiere le imprese di trasformazione di piccolissima dimensione sono integrate a monte con il sistema agricolo.

Le principali tipologie di innovazioni introdotte sono quelle di prodotto $(68,8 \%)$ e, in misura meno rilevante, quelle di processo (50\%) (Tabella 6). Per quanto riguarda le prime, le imprese del cluster hanno dichiarato che la realizzazione di nuovi prodotti è volta a soddisfare soprattutto i nuovi trend di consumo. Nel caso delle imprese viti-vinicole, un esempio è rappresentato dalla produzione di nuovi vini senza solfiti e lieviti o dalle nuove linee di vino spumante. Le innovazioni di prodotto nella altre filiere riguardano soprattutto la realizzazione di prodotti sostenibili e con packaging attivi oltre che una maggiore completezza dell'etichettatura, tale da garantire la tracciabilità dei prodotti. Le innovazioni di processo hanno riguardato invece l'utilizzo di macchinari di agricoltura di precisione con sensori multi spettrali o la realizzazione di impianti fotovoltaici per il fabbisogno energetico aziendale. Riguardo alle innovazioni di marketing $(43,8 \%)$, le imprese del cluster si sono focalizzate principalmente sulla realizzazione di cambiamenti significativi nell'attività di vendita e distribuzione e nel packaging.

Questo cluster può essere considerato "con alto potenziale innovativo", poiché le innovazioni sono state realizzate utilizzando prevalentemente risorse interne e sfruttando relativamente poco il ricorso alle collaborazioni. A tale riguardo va, infatti, considerato che le nuove tecnologie nascono solitamente in settori profondamente diversi da quello agroalimentare, per cui lo sviluppo di rapporti di collaborazione con soggetti esterni a spiccata vocazione innovativa e tecnologica, può favorire il contenimento dei costi di sviluppo interno ed accelerare i tempi di realizzazione ed immissione dei nuovi prodotti sul mercato.

\subsubsection{Le imprese Non Innovative (NI)}

Il terzo cluster, rappresentato dalle imprese Non Innovative (NI), contiene il più alto numero di imprese del campione (56,5\%) ed è costituito da due sottogruppi che attribuiscono una differente importanza ai fattori di
Marcella De Martino Fabio Magnotti Lodovico Santoro L'innovazione nelle piccole e medie imprese agroalimentari della Regione Campania 
sinergie Vol. 36, N. 105, 2018

ostacolo allinnovazione: Microimprese Non Innovative (MNI) e Piccole e Medie Imprese Non Innovative (PMINI). Il sottogruppo MNI rappresenta il $35,1 \%$ del campione ed è composto esclusivamente da microimprese con meno di 10 addetti e con un fatturato inferiore a $€ 250.000$ (Tabella 4). Il PMINI rappresenta invece il $21,4 \%$ del campione ed è composto soprattutto da piccole imprese (88\%) con un numero di addetti compreso tra 10 e 49 ed un fatturato più elevato delle MNI.

Un elemento comune ad entrambi i sottogruppi è rappresentato dalla forte incidenza di casi che hanno dichiarato di non aver mai esportato i propri prodotti all'estero, sebbene tale tendenza sia maggiore tra le MNI (70,7\%). Dall'indagine risulta, altresì, che nessuna delle imprese del cluster ha partecipato a programmi di finanziamento europeo, nazionale o regionale, non sfruttando così i vantaggi associabili all'utilizzo di risorse esterne quali i finanziamenti pubblici per l'innovazione.

Nella Figura 2 sono riportati i principali fattori di ostacolo ai processi innovativi. In particolare è possibile osservare come per le microimprese (MNI) il fattore di ostacolo più rilevante sia costituito dalla presenza di imprese leader nel proprio mercato di riferimento (85\%). Questa percezione si può spiegare considerando il ruolo delle microimprese nell'ambito della filiera, le quali operando prevalentemente come subfornitori della Grande Distribuzione Organizzata (GDO), hanno una compressione dei margini di profittabilità. Questo fenomeno è aggravato spesso dalla mancanza di una struttura organizzativa in grado di sostenere politiche di penetrazione commerciale verso il sistema distributivo e/o il consumatore finale.

Fig. 2: Ostacoli all'innovazione (frequenze \%)

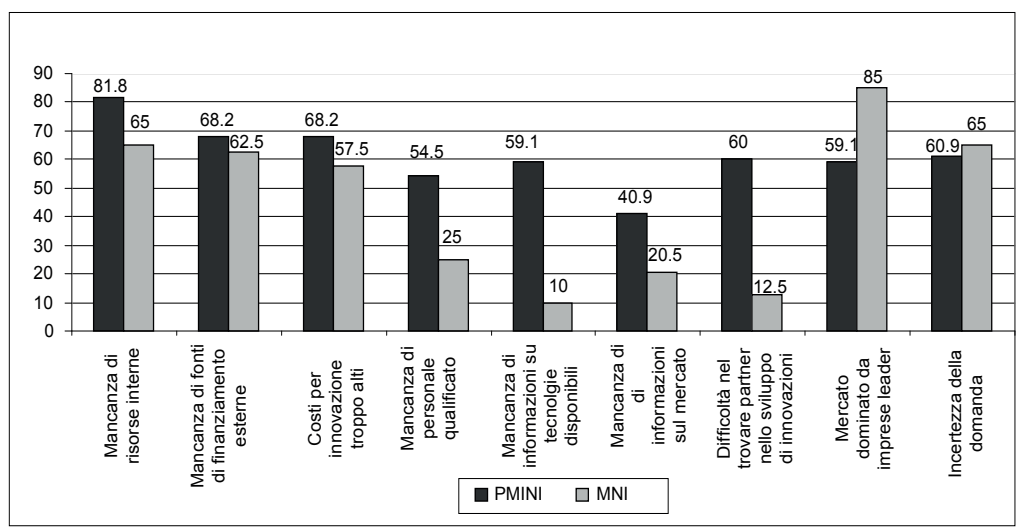

Fonte: nostra elaborazione

Inoltre, la mancanza di risorse interne (65\%), la carenza di finanziamenti esterni $(62,5 \%)$ e i costi per innovazione troppo alti $(57,5)$ rappresentano i principali fattori di ostacolo allinnovazione. Tali fattori risultano legati principalmente alla struttura finanziaria di queste microimprese, che dispongono di risorse limitate da investire in attività di R\&S. I fattori di natura economico-finanziaria sono d'altronde considerati come uno dei principaliostacoliall'innovazione anche nel tessuto imprenditoriale italiano. 
In tal senso, come suggerisce Giaretta (2013) il costo per l'innovazione non deve essere considerato limitatamente al periodo di sviluppo ma anche e soprattutto a quello di diffusione delle innovazioni, che generalmente impegna l'azienda per un maggiore e indefinito orizzonte temporale. Tale circostanza incide notevolmente sull'esposizione finanziaria delle imprese, soprattutto quelle di piccole dimensioni, spingendo spesso progetti innovativi ad arenarsi, nonostante le elevate potenzialità di sviluppo.

Con riferimento alle imprese del secondo sottogruppo (PMINI), l'elemento più rilevante che ostacola gli investimenti in attività di R\&S è rappresentato dalla carenza di personale qualificato $(54,5 \%)$ e dalle insufficienti risorse interne, sia finanziarie che tecnologiche $(81,8 \%)$. Tali imprese, infatti, ritengono fondamentale il possesso di queste risorse soprattutto qualora lo sviluppo di una nuova tecnologia o prodotto risulti eccessivamente oneroso. Un ulteriore ostacolo all'innovazione per le PMINI è rappresentato dall'elevato costo dell'innovazione $(68,2 \%)$. Tale risultato è dovuto principalmente all'impossibilità di trasferire i benefici connessi ai risultati delle attività di R\&S sui volumi di produzione, i cui livelli sono tali da non consentire la riduzione dellincidenza del costo dell'innovazione sul prezzo finale del prodotto.

Infine, un fattore percepito quale ostacolo all'innovazione da entrambi i sottogruppi di imprese (65\% delle MNI e dal 60,9\% delle PMINI) è rappresentato dall'incertezza sull'andamento della domanda che, soprattutto in fasi congiunturali negative, può scoraggiare l'attività di innovazione anche di imprese che avrebbero la possibilità di farlo.

\subsection{I risultati dell'innovazione}

Un aspetto interessante della ricerca riguarda l'analisi degli effetti dell'innovazione che consente di comprendere i risultati che le imprese innovatrici hanno ottenuto nel triennio 2011-2013.

Con riferimento alle imprese del primo cluster (IC), quest'ultime percepiscono tra i maggiori benefici l'ampliamento della capacità produttiva $(62,5 \%)$, la maggiore capacità di conformarsi alla normativa $(62,5 \%)$, l'ingresso in nuovi mercati $(60 \%)$ e la riduzione dell'impatto ambientale $(58,8 \%)$ (Figura 3 ). Lampliamento della capacità produttiva permette alle imprese di conseguire economie di scala che danno luogo ad una riduzione dei costi unitari. Il conseguente aumento dei volumi di produzione associato ad una maggiore efficienza produttiva può incentivare l'espansione dell'impresa, facilitando la penetrazione in nuovi mercati altamente competitivi.

Ladempimento delle normative relative al settore agroalimentare, invece, è il risultato del consolidamento dei rapporti di tali imprese con il settore pubblico. Oltre ai finanziamenti, tali rapporti riguardano anche l'adeguamento alle politiche europee e nazionali sia in materia di certificazioni di qualità dei prodotti agroalimentari (DOP e IGP) sia in materia di sostenibilità delle produzioni. In maniera simile, la riduzione degli impatti ambientali, percepita come beneficio raggiunto tramite l'innovazione dal 58,8\% delle IC, è legato all'adempimento delle normative comunitarie in materia di sostenibilità ambientale (emissioni di $\mathrm{CO}_{2}$, uso Marcella De Martino
Fabio Magnotti Lodovico Santoro Linnovazione nelle piccole e medie imprese agroalimentari della Regione Campania 
sinergie

italian journal of management Vol. 36, N. 105, 2018

di nitrati, pesticidi, nonché protezione del suolo, gestione delle risorse idriche e conservazione delle biodiversità). Tali risultati vanno letti congiuntamente con il trend positivo registrato dal fatturato delle IC che, rispetto agli altri cluster, presentano nella maggior parte dei casi $(73,7 \%)$ un fatturato in crescita, nonostante la crisi economica che caratterizzava il periodo dell'indagine (Figura 4).

Nonostante le ridotte dimensioni aziendali che caratterizzano le imprese del secondo cluster (INC), le innovazioni da queste adottate hanno tuttavia condotto a risultati aziendali positivi. In particolare, laumento dell'offerta dei prodotti $(72,2 \%)$ è percepito come il beneficio maggiormente associato all'innovazione (Figura 3).

Fig. 3: Risultati derivanti dall'introduzione di innovazioni

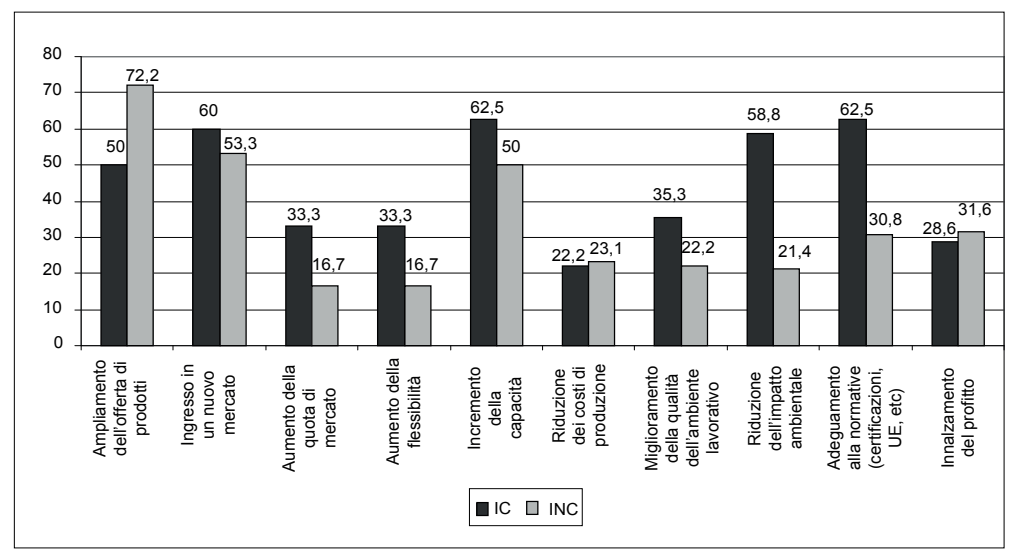

Fonte: nostra elaborazione

Tale tendenza è da collegare principalmente alle innovazioni di prodotto e di marketing, le quali permettono di ottenere un vantaggio competitivo attraverso l'introduzione di nuovi prodotti sul mercato. Congiuntamente, va considerato che l'ingresso in nuovi mercati rappresenta il secondo risultato percepito come più importante dalle INC $(53,3 \%)$. Tale fattore rappresenta unopportunità, non solo per aumentare i propri ricavi di vendita e rafforzare il proprio vantaggio competitivo, ma anche per instaurare nuovi rapporti di collaborazione e aumentare le proprie conoscenze utili per l'attivazione di ulteriori processi innovativi.

Anche l'aumento della capacità produttiva è percepito come un risultato importante da molte imprese del cluster (50\%). Questo può condurre, a sua volta, ad un aumento dei volumi di fatturato attraverso cui finanziare ulteriori progetti di innovazione. Nonostante le ridotte dimensioni, l'approccio aziendale orientato alle innovazioni ha permesso alle INC di ottenere un trend del fatturato positivo o stabile, rispettivamente nel 34,4\% e nel 53,1\% dei casi (Figura 4). 
Fig. 4: Trend di fatturato (2010-2013) (frequenze \%)

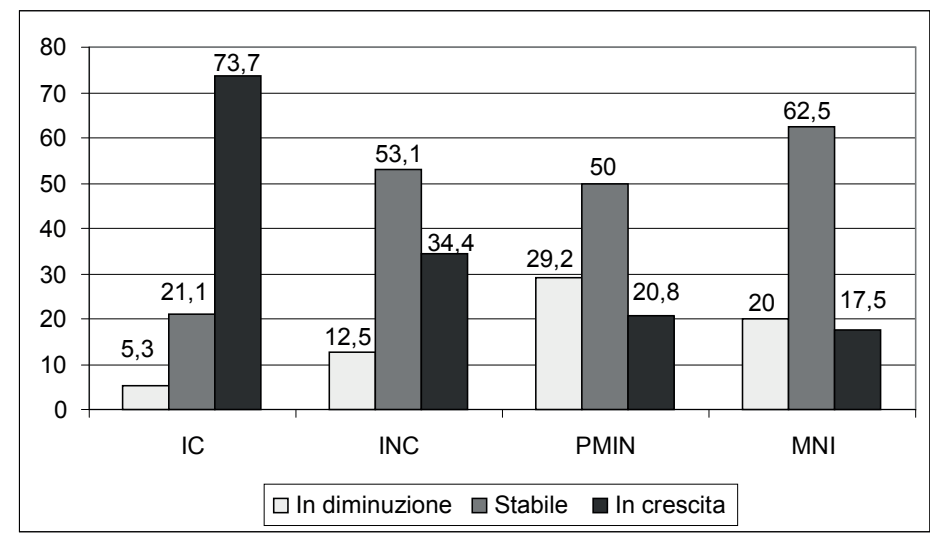

Marcella De Martino

Fabio Magnotti

Lodovico Santoro

L'innovazione nelle

piccole e medie imprese

agroalimentari della

Regione Campania

Fonte: nostra elaborazione

\section{Conclusioni e future direzioni della ricerca}

Lanalisi svolta ha permesso di identificare tre gruppi di imprese, due dei quali (IC e INC) hanno intrapreso processi innovativi con diversa intensità di utilizzo delle risorse interne ed esterne, mentre il terzo gruppo (NI) non ha realizzato alcuna innovazione.

In particolare, il primo gruppo delle imprese innovative ha beneficiato del rapporto di collaborazione istaurato con i fornitori di materie prime e di macchinari e software. Questo risultato è in linea con quanto ampiamente dimostrato dalla letteratura che considera i rapporti con gli attori della supply chain una condizione necessaria per realizzare con successo le innovazioni, specialmente nel caso di prodotti con nuove caratteristiche qualitative. Rispetto alla totalità delle imprese intervistate, però, tale pratica risulta essere ancora poco diffusa, in quanto le aziende appartenenti a tale cluster rappresentano solo il $16,2 \%$ dell'intero campione. Due sono gli elementi critici che possono offrire alcuni spunti di riflessione affinché sia possibile favorire la diffusione di una cultura dell'innovazione nelle PMI, sia nell'ambito della supply chain che nel contesto territoriale di riferimento.

Un primo punto critico per lo sviluppo e diffusione dell'innovazione è rappresentato dalla necessità che i vari attori della supply chain e gli stakeholder locali (come ad esempio Università, enti di ricerca) condividano un patrimonio cognitivo comune, il che non è realizzabile senza la mediazione di un'identità e di valori condivisi. La fiducia è un fattore chiave per il successo di molte reti inter-organizzative che, spesso, necessitano di appropriate forme di governo al fine di prevenire comportamenti opportunistici da parte degli altri attori della rete. Il decisore pubblico può svolgere, a tal riguardo, un ruolo chiave nel promuovere e incentivare, anche attraverso forme di sostegno alla collaborazione pubblico-privata, lo sviluppo di reti inter-organizzative.

Un secondo punto critico è legato alla necessità di diffondere una cultura all'innovazione presso le microimprese campane, spesso restie ad attivare 
sinergie Vol. 36, N. 105, 2018

processi di cambiamento organizzativo o ad aprire i confini aziendali ad altri attori esterni. I dati relativi al gruppo delle imprese IC mostrano che esse hanno ottenuto risultati aziendali migliori rispetto alle altre imprese appartenenti ai cluster INC e NI. La diffusione di "best practice" potrebbe incentivare, a tal riguardo, l'adozione di pratiche collaborative da parte di queste imprese, anche in virtù del fatto che solitamente esse innovano per imitazione.

Un ulteriore risultato è che la capacità di sapersi avvalere delle opportunità offerte dai finanziamenti pubblici può favorire i processi innovativi delle PMI agroalimentari. L'indagine, infatti, ha mostrato che la quasi totalità delle imprese del cluster IC ha beneficiato di finanziamenti pubblici per la realizzazione di attività di innovazione, soprattutto di provenienza regionale e/o nazionale. L'utilizzo di fondi europei è, però, molto ridotto; del tutto assente nel caso delle microimprese del cluster INC. Le fonti di finanziamento pubblico possono rappresentare, a tal riguardo, importanti strumenti di incentivo all'innovazione, beneficiando anche delle collaborazioni a livello europeo, qualora le imprese siano informate ed assistite nella loro partecipazione alle varie iniziative progettuali. A questo proposito, alcune di esse hanno identificato nelle Associazioni di categoria il soggetto chiave in grado di supportale nell'identificazione dei bandi più appropriati. Ciò risulta essere ancor più necessario per le micro imprese del cluster MNI, che identificano nella presenza di un leader di settore il principale ostacolo allinnovazione e, pertanto, sono disincentivate a partecipare a progetti comuni, basandosi sulla percezione di avere un peso relativo minore nellambito della partnership.

Dall'analisi emerge, anche, l'importanza degli investimenti in attività di R\&S nel favorire l'adozione di processi innovativi, sebbene la natura stessa dell'innovazione nelle PMI sia spesso legata a fattori difficilmente rilevabili formalmente, tanto da essere stata definita "invisibile". Difatti, la presente ricerca conferma la presenza di PMI che hanno saputo innovare attraverso la partecipazione a network collaborativi e/o la formazione dei lavoratori finalizzata allo sviluppo o all'introduzione di innovazioni di prodotto o di processo, nonché attraverso le attività di marketing e advertising, di supporto alla vendita di nuovi prodotti. A tal riguardo, il presente lavoro propone un set di indicatori in grado di cogliere la capacità innovativa delle PMI, in linea con il framework adottato nelle indagini CIS, pertanto adatto a fornire risultati comparabili ed indicazioni di policy a livello locale. Il valore aggiunto risiede sia nellaver saputo coinvolgere realtà di piccola e piccolissima dimensione, spesso restie alla partecipazione ad indagine empiriche, sia nell'aver esplorato la natura e le tipologie di innovazione da esse realizzate negli ultimi tre anni. Le Associazioni di categoria risultano, a tal riguardo, un soggetto chiave per il coinvolgimento delle PMI e per lefficace somministrazione del questionario in sede aziendale.

Il lavoro fornisce, in conclusione, una analisi approfondita dei fattori che influenzano la capacità innovativa delle piccole e medie imprese nel settore agro-alimentare. Tuttavia, sono presenti alcuni limiti che la futura ricerca dovrebbe superare. In particolare il framework utilizzato offre un quadro completo ma descrittivo dei fattori che facilitano l'innovazione nelle PMI. A tal riguardo, la realizzazione di un'indagine longitudinale 
di alcune best practice di piccole imprese innovative consentirebbe di analizzare i processi di cambiamento e gli effetti di lungo termine prodotti dalle innovazioni che per loro natura non sono immediati. In tal modo sarebbe possibile svolgere analisi più approfondite sul nesso di causalità tra fattori (driver) e risultati ottenuti attraverso i processi innovativi. Inoltre, alcuni aspetti legati all'impresa a gestione familiare tipica delle PMI - che rappresenta l'ossatura del sistema imprenditoriale italiano - meritano attenzione. In particolare, le imprese familiari presentano solitamente una minore propensione al rischio, quale conseguenza della sostanziale coincidenza tra patrimonio familiare e patrimonio di impresa. Si potrebbe investigare il ruolo che la natura familiare della proprietà svolge nel facilitare od ostacolare lo sviluppo di processi innovativi, approfondendo anche gli effetti della trasmissione intergenerazionale delle imprese. L'analisi delle caratteristiche dell'imprenditore innovatore, inoltre, e dei diversi canali attraverso cui influenza ed è influenzato dal sistema tecnico, economico e sociale che lo circonda (Gambardella, 2014), offrirebbe un ulteriore elemento critico di riflessione sul tema dell'innovazione invisibile, che sfugge alle rilevazioni statistiche nazionali.

I risultati conseguiti nell'ambito della ricerca sono stati discussi con le principali Associazioni di categoria, al fine di delineare azioni volte a favorire una maggiore diffusione dell'innovazione nell'ambito delle filiere agroalimentari e dell'intero territorio campano. Si riconosce la necessità di adottare un approccio sistemico di sviluppo dell'innovazione che passi attraverso il consolidamento del cosiddetto "sistema innovativo regionale", formato dall'insieme dei soggetti, delle funzioni e delle azioni interessate ai meccanismi di diffusione della conoscenza e dell'innovazione. Attivare l'innovazione e governare il sistema della conoscenza, deve essere uno stimolo per perseguire una più generale eccellenza non solo d'impresa, ma di sistema sull'intero territorio interessato a queste produzioni. Questo richiede, difatti, un rafforzamento del partenariato, ed in particolare la promozione dell'approccio c. d. "a tripla elica", secondo il quale è necessario realizzare una stretta interazione tra il mondo della ricerca, dell'impresa e della pubblica amministrazione, allo scopo di favorire le più adeguate sinergie e complementarietà tra le diverse politiche e gli strumenti finanziari connessi.

\section{Bibliografia}

ACOSTA M., CORONADO D., ROMERO C. (2015), "Linking public support, $\mathrm{R} \& \mathrm{D}$, innovation and productivity: New evidence from the Spanish food industry", Food Policy, vol. 57, pp. 50-61.

AMBROSETTI CLUB (2013), L'ecosistema per l'innovazione: quali strade per la crescita delle imprese e del Paese, The European House, Technology Forum.

AVERMAETE T., VIAENE J., MORGAN E.J., PITTS E., CRAWFORD N., MAHON D. (2004), "Determinants of product and process innovation in small food manufacturing firms", Trends in food science and technology, vol. 15 , n. 10 , pp. $474-483$.

BANCA D'ITALIA (2012), "Il gap innovativo del sistema produttivo italiano: radici e possibili rimedi”, Questioni di Economia e Finanza, n. 121, Aprile 2012.
Marcella De Martino

Fabio Magnotti

Lodovico Santoro

L'innovazione nelle

piccole e medie imprese

agroalimentari della

Regione Campania 
BAREGHEH A., ROWLEY J., SAMBROOK S., DAVIES D. (2012), "Innovation in food sector SMEs", Journal of Small Business and Enterprise Development, vol. 19, n. 2, pp. 300-321.

BATTERINK M., WUBBEN E., OMTA S. (2006), "Factors related to innovative output in the Dutch agrifood industry", Journal on Chain and Network Science, vol. 6, n. 1, pp. 31-44.

BATTERINK M.H., WUBBEN E.F., KLERKX L., OMTA S.W.F. (2010), "Orchestrating innovation networks: The case of innovation brokers in the agri-food sector", Entrepreneurship and Regional Development, vol. 22, n. 1, pp. 47-76.

BOERMANS M.A., ROELFSEMA H. (2016), "Small firm internationalization, innovation, and growth", International Economics and Economic Policy, vol. 13, n. 2, pp. 283-296.

CAPITANIO F., COPPOLA A., PASCUCCI S. (2009), "Indications for drivers of innovation in the food sector", British Food Journal, vol. 111, n. 8, pp. 820838.

CHIU T., FANG D., CHEN J., WANG Y., JERIS C. (2001), "A robust and scalable clustering algorithm for mixed type attributes in large database environment", in Proceedings of the seventh ACM SIGKDD International conference on knowledge discovery and data mining, pp. 263-268.

COLOMBO M.G., LAURSEN K., MAGNUSSON M., ROSSI-LAMASTRA C. (2012), "Small business and networked innovation: organizational and managerial challenges", Journal of Small Business Management, vol. 50, n. 2, pp. 181-190.

CRÉPON B., DUGUET E., MAIRESSE J. (1998), 'Research and Development, Innovation and Productivity: An Econometric Analysis at the Firm Level”, Economics of Innovation and New Technology, vol. 7, n. 2, pp. 115-158.

DAGNINOG.B.,LEVANTIG., MINÀA.,PICONEP.M.(2015), "Interorganizational network and innovation: a bibliometric study and proposed research agenda", Journal of Business and Industrial Marketing, vol. 30, n. 3/4, pp. 354-377.

DAVENPORT S. (2005), "Exploring the role of proximity in SME knowledgeacquisition”, Research Policy, vol. 34, pp. 683-701.

DE JONG P.J., VERMEULEN P.A.M. (2006), "Determinants of product innovation in small firms: a comparison across industries", International Small Business Journal, vol. 24 n. 6, pp. 587-609.

DE MARTINO M., MAGNOTTI F. (2018), “The innovation capacity of small food firms in Italy”, European Journal of Innovation Management, https://doi. org/10.1108/EJIM-04-2018-0041

DE MARTINO M., MAGNOTTI F., VOLPE T. (2014), "Analisi delle filiere agroalimentari", Rapporto di ricerca del progetto Campus innovazione QUARC "Qualità delle produzioni tipiche campane ed il suo territorio: approcci innovativi ed integrati per rafforzare la competitività del sistema Agroalimentare.

EUROPEAN COMMISSION (2003), Commission Recommendation 2003/361/EC.

EUROSTAT (2014), Community Innovation Survey 2014, The statistical office of the European Union, Brussels.

GAMBARDELLA A. (2014), "Limprenditore innovatore come agente dello sviluppo tecnico, economico e sociale”, Sinergie, n. 93, pp. 3-18. 
GIARETTA E. (2013), "Linnovazione aziendale: aspetti concettuali e approcci gestionali", in Baccarani C., Brunetti F., Giaretta E. (a cura di), Impresa e management tra competitività e progresso, Giappichelli, Torino.

GRONUM S., VERREYNNE M.L., KASTELLE T. (2012), “The role of networks agroalimentari della in small and medium-sized enterprise innovation and firm performance", Journal of Small Business Management, vol. 50, n. 2, pp. 257-282.

GRUNERT K.G., HARMSEN H., MEULENBERG M., KUIPER E., OTTOWITZ T., DECLERCK F., GÖRANSSON G. (1997), "A framework for analysing innovation in the food sector", in Products and Process Innovation in the Food Industry, Springer US, pp. 1-37.

ISTAT (2011), $9^{\circ}$ Censimento generale dell'Industria e dei Servizi, Roma.

KANTER R.M. (1994), "Collaborative advantage: the art of alliances", Harvard Business Review, vol. 72, n. 4, pp. 96-108.

KARANTININIS K., SAUER J., FURTAN W.H. (2010), "Innovation and integration in the agri-food industry", Food Policy, Vol. 36, n. 2, pp. 112-120.

KÜHNE B., VANHONACKER F., GELLYNCK X., VERBEKE W. (2010), "Innovation in traditional food products in Europe: Do sector innovation activities match consumers' acceptance?", Food Quality and Preference, vol. 21, n. 6, pp. 629-638.

LAZZERONI M. (2010), "High-tech activities, system innovativeness and geographical concentration: Insights into technological districts in Italy", European Urban and Regional Studies, vol. 17, n. 1, pp. 45-63.

LUNDVALL B. (2010), National systems of innovation: Toward a theory of innovation and interactive learning, Pinter Publishers, London.

MASCITELLI R. (2000), "From experience: harnessing tacit knowledge to achieve breakthrough innovation", Journal of Product Innovation Management, vol. 17, n. 3, pp. 179-193.

MIPAAF (2016), Elenco delle denominazioni italiane, iscritte nel Registro delle denominazioni di origine protette, delle indicazioni geografiche protette e delle specialità tradizionali garantite (Regolamento UE $n$ 1151/2012 del Parlamento europeo e del Consiglio del 21 novembre 2012), Roma. https://www.politicheagricole.it/flex/cm/pages/ServeBLOB.php/L/IT/ IDPagina/2090

MOOI E., SARSTEDT M. (2011), A Concise Guide to Market Research: The Process, Data, and Methods Using IBM SPSS Statistics, Springer, Berlin, NY.

ÖZÇELIK E., TAYMAZ E. (2004), "Does innovativeness matter for international competitiveness in developing countries? The case of Turkish manufacturing industries", Research Policy, vol. 33, n. 3, pp. 409-424.

SANGUIGNI V., DE CRESCENZO E. (2013), "Lo sviluppo delle potenzialità del territorio attraverso i distretti tecnologici. Un'analisi desk della Regione Campania", in L'innovazione per la competitività delle imprese, Refferred Electronic Conference Proceedings, XXV Convegno Annuale di Sinergie, Università Politecnica delle Marche, Ancona, 24-25 ottobre.

SANTAMARÍA L., NIETO M.J., BARGE-GIL A. (2009), "Beyond formal R\&D: Taking advantage of other sources of innovation in low-and mediumtechnology industries", Research Policy, vol. 38, n. 3, pp. 507-517.

SHAN P., SONG M., JU X. (2016), "Entrepreneurial orientation and performance: is innovation speed a missing link?", Journal of Business Research, vol. 69, n. 2, pp. 683-690. 


\section{sinergie} Vol. 36, N. 105, 2018

THE EUROPEAN HOUSE - AMBROSETTI, FEDERALIMENTARI (2018), "Sostenere la crescita di lungo periodo e l'internazionalizzazione delle imprese del settore food \& beverage in Italia", Rapporto Cibus connect.

VON HIPPEL E. (1988), The Sources of Innovation, Oxford University Press, Oxford.

\section{Academic or professional position and contacts}

\section{Marcella De Martino}

Researcher in Management

Istituto di Ricerca su Innovazione e Servizi per lo Sviluppo (IRISS) - CNR - Napoli - Italy e-mail: m.demartino@iriss.cnr.it

\section{Fabio Magnotti}

Research Fellow in Management

Istituto di Ricerca su Innovazione e Servizi per lo Sviluppo (IRISS) - CNR - Napoli - Italy e-mail: f.magnotti@iriss.cnr.it

\section{Lodovico Santoro}

Research Assistant in Economics and Statistics

Istituto di Ricerca su Innovazione e Servizi per lo Sviluppo (IRISS) - CNR - Napoli - Italy e-mail: 1.santoro@iriss.cnr.it 